possibilities; if the restoration is replaced, it is considered to have failed or the restoration has failed when the decision to replace it was based on clearly defined guidelines.

The lack of controlled clinical trials shows the difficulties of carrying out these trials in practice and therefore, throws into question the motivation in the decisions made by the practitioners who carried out the treatments reported on in this paper.

There are several factors that contribute to prescribing patterns:

- Undergraduate or postgraduate training

- Time available for treatments

- Financial pressures

- Gender

- Age

- The environment the practitioner is working in be it general practice, hospital or academic.

All of these will influence, more or less, the choice or preference for treatment.

Daniel Kahneman, in his book Thinking, fast and slow ${ }^{2}$ writes: 'The extent of consistency is often a matter of concern' and mentions how experienced radiologists who evaluate chest $\mathrm{x}$-rays as 'normal' or 'abnormal' contradict themselves some of the time when they see the picture on different occasions.

As human beings we are not as consistent or reliable as we would like to think. And how useful is this information?

With the patient sitting in the dental chair, the question that confronts the dentist is 'what do I do here?' The practitioner has to make a decision within the context of the patient. If the tooth were out of the mouth and held in the hand the 'best' way to bring it to form and function may be one type of treatment.

But, in the context of the mouth, modifying factors have to be taken into account and the option for 'best' treatment may be quite different.

The real challenge is for practitioners to be aware of all the treatment options and how and why they arrive at the option of choice and to be conscious of the question as to whose interests are being served in the provision of treatment. Is it the patient's or the dentist's? Hopefully, both!

R. Caplin, by email

1. Burke F J T, Lucarotti P S K. The ultimate guide to restoration longevity in England and Wales. Part 5: crowns: time to next intervention and to extraction of the restored tooth. Br Dent J 2018; 225: 33-48.

2. Kahneman D. Thinking, fast and slow. p 225. Penguin Books, 2012.

DOI: $10.1038 /$ sj.bdj.2018.763
Dental research

\section{International research collaboration}

Sir, the office of the International Association for Dental Research Caribbean Section [IADR-CS] is pleased to share the official launch of its Newsletter.

The overall objectives are strengthening the mission and vision of IADR among Caribbean members, providing an opportunity for collaboration and the promotion and dissemination of research activities from dentists and specialist dentists within the region.

The launch of the first issue took place on 15 June 2018 at the IADR-CS meeting: the 3rd Caribbean Oral Health Initiative Summit in Puerto Rico, USA.

The contents of the newsletter include a message from the IADR President, key events, and research at Trinidad and Tobago. The full text is available at https://goo.gl/XgPUQh.

We continue to look forward to research collaboration across global dental researchers.

\section{A. B. R. Santosh, J. Collins, L. Feliz and \\ N. Abreu, by email \\ DOI: 10.1038/sj.bdj.2018.764}

\section{Dental trauma}

\section{Trauma protocol for schools}

Sir, a recent case in practice has led me to carry out a service evaluation in the local community - assessing the knowledge of the management of dental trauma amongst school teachers in the North East.

A nine-year-old girl attended with a complicated enamel-dentine fracture 11 and an uncomplicated enamel-denture fracture on the 21 , which occurred during lunch time. She attended one hour after the incident with her teacher, who was also her mother.

On review of the incident with the child's mother, it was reported that there was no dental emergency protocol at the school.

A brief 'trial' survey distributed to the schools near the practice showed that on review of 45 responses from the teachers and staff within these schools, there was an overall unsatisfactory knowledge of the appropriate management procedures and protocol a school could have in place to prevent, manage and treat the various dental injuries which may occur during school hours.

The Department for Education has confirmed that its current health and safety requirements for schools lie in the hands of the school's owner/employer (usually the local council), and they should ensure there are appropriate measures in place so that the safety of staff and pupils are adequate. When it comes to dental trauma and its management, it seems there is no such appropriate measure in place yet.

I have distributed a region-wide voluntary questionnaire to all the schools in the North East, for assessment of their knowledge and desire to seek an appropriate method of training and advice.

Post evaluation of this data, I aim to provide a dental trauma protocol for schools and a 'training package' for teachers, which I am currently constructing.

I also plan to submit a similar letter to an education journal in the North East to increase awareness of my project and enthuse schools to take part.

G. O'Neill, by email DOI: 10.1038/sj.bdj.2018.765

\section{Occlusion}

\section{Time to debate malocclusion}

Sir, I believe dentistry could be at the centre of a health revolution. There is the very real possibility that simple public health measures could minimise and even eliminate malocclusion, TMJ and sleep apnoea, thereby improving the lives of countless individuals and saving the NHS huge sums of money. I don't have all the answers, but I am asking the right questions. I want the truth, backed by scientific evidence, so our patients can benefit.

For a decade, I have raised concerns that our profession treats a modern disease, malocclusion, as a genetic inevitability. The evidence for an environmental origin is unambiguous. Less than 1,000 years ago, the vast majority of people gained and maintained all of their teeth in reasonable occlusions and alignment from birth to death.

Today, more than a third of 12-yearold children in the UK have an index of treatment need score of 3.8 or above and permanent retention is routine.

We should work together to change this by finding definitive answers to questions such as:

- Why has there been such a rapid rise in malocclusion?

- Why do teeth become crooked in the first place?

- How is TMD related to malocclusion?

- Will root resorption affect root canal therapy? 